\title{
Feasibility of dc vaccine combined with low dose endoxan and high dose il-2 treatment associated with taurolidine for advanced, multi-resistant melanoma patients.
}

\author{
Gustavo A Moviglia ${ }^{*}$, Ralf Kleef ${ }^{2}$
}

From 30th Annual Meeting and Associated Programs of the Society for Immunotherapy of Cancer (SITC 2015)

National Harbor, MD, USA. 4-8 November 2015

\section{Background}

2014 Dillman and coworkers reported 40\% 5-year survival of advanced malignant melanoma (MM) patients treated with a combination of Tumor Vaccine in combination with High dose IL-2 versus $13 \%$ of similar patients treated only with High Dose of IL-2 (HD IL-2). In order to improve the efficacy of this approach and minimize the adverse effects of HD IL-2 therapy we have developed a protocol using a Dendritic Cell Vaccine challenged with autologous MM stem cells in combination with HD IL-2 and to Taurolidine (to diminish the vasculary leak syndrome).

\section{Methods}

5 advanced and rapidly progressive MM patients, resistant to any other standard therapy were treated. $1 / 5$ with large brain metastasis was withdrawn from the treatment after the first cycle of treatment for rapid progression of his disease. 4/5 patients received three cycles. In brief, previous to vaccination with a patient specific autologous dendritic cell vaccine patients underwent low dose endoxan $300 \mathrm{~m} / \mathrm{m} 2$, and were subsequently treated for 5 days with high-dose IL-2 in combination with Taurolidine as described by O'Brian et.al. 2006.

\section{Results}

Major side effects were high temperature $4 / 4$ and hypereosinophilia associated with itching (2/5). No other signs like severe edema, renal failure or any other life threatening condition was observed. 4/5 patients did not show any progression of their condition during the 2-3 months of observation.

\section{Conclusions}

DC-vaccine associated to HD IL-2 + Taurolidine vaccine seems to be a feasible and low toxic treatment. Longer observation time as well as increment of the number of patients treated is necessary to confirm these preliminary findings.

\section{Authors' details \\ ${ }^{1}$ Maimonides University, Ciudad de Buenos Aires, Argentina. ${ }^{2}$ Maimonides \\ University, Vienna, Austria.}

Published: 4 November 2015

doi:10.1186/2051-1426-3-S2-P209

Cite this article as: Moviglia and Kleef: Feasibility of dc vaccine

combined with low dose endoxan and high dose il-2 treatment

associated with taurolidine for advanced, multi-resistant melanoma patients.. Journal for ImmunoTherapy of Cancer 2015 3(Suppl 2):P209.

'Maimonides University, Ciudad de Buenos Aires, Argentina

Full list of author information is available at the end of the article 